\title{
Proactive and Reactive DF Relaying for Cognitive Network with Multiple Primary Users
}

\author{
Chanchal Kumar DE ${ }^{1}$, Sumit KUNDU ${ }^{2}$ \\ ${ }^{1}$ Electronics \& Instrumentation Engineering Department, Dr. B. C. Roy Engineering College, Durgapur 713206, WB, India \\ ${ }^{2}$ Electronics \& Communication Engineering Department, National Institute of Technology, Durgapur 713209, WB, India
}

wrt2chanchal@ rediffmail.com, sumit.kundu@ece.nitdgp.ac.in

Manuscript received September 29, 2015

\begin{abstract}
In this paper, the outage performance of a cognitive radio network with a pair of secondary transmitter and receiver is investigated in the presence multiple primary users over a number of licensed frequency band and multiple secondary relays (SRs). Two decode and forward (DF) schemes are considered for the relays, namely proactive and reactive DF schemes. An adaptive power allocation scheme for secondary transmitter and secondary relays is formulated under the joint constraints of the primary user outage and peak transmit power of the secondary users. Based on these strategies, analytical expressions for the outage probability of proactive and reactive DF schemes are derived. More precisely, our results demonstrate the impact of number of the active primary users (PUS) over a number of available licensed frequency bands on the outage performance of secondary network. Further, it is observed that the performance of the secondary network can be improved by extending the bandwidth of the primary users.
\end{abstract}

\section{Keywords}

Cognitive radio network, proactive and reactive DF, Secondary User (SU), peak transmit power

\section{Introduction}

Recently, cognitive radio technology has been proposed to alleviate the inefficient usage of radio frequency spectrum in wireless network [1]. In particular, under spectrum sharing environment, the secondary user (SU) is allowed to access the radio frequency band as long as its transmit power is maintained below a given threshold which is imposed by the interference power constraint at the PU receiver [2]. At the same time, the relaying transmission has been demonstrated as an effective means to boost the throughput and extend the coverage of wireless communication system [3-6]. Hence, investigating the performance of relaying transmission in spectrum sharing system and extension of opportunistic relay selection to cognitive network have attracted great interest [7-11].
Specifically, closed-form expressions of outage probability (OP), symbol error probability (SEP) and ergodic capacity for opportunistic AF relaying over non identical Rayleigh fading channels have been presented [7]. An asymptotic expression for opportunistic relaying under spectrum-sharing approach has been derived [8], which shows that the diversity gain is equal to the number of cognitive relays. Very recently, the performance of cognitive opportunistic relaying network over frequency selective fading channel has been reported [9]. The joint outage constraint of the PU and peak transmit power constraint of the SU on the outage performance of a cognitive cooperative radio network (CCRN) using the proactive and reactive DF schemes has been investigated in [10]. The outage probability and power allocation for the two-way decode-and-forward (DF) relay network with relay selection has been investigated in [11]. The outage probability of a simple dual-hop cooperative spectrum-sharing systems (CSSS) with an interference constraint on PU in a Nakagami- $m$ fading channel has been analyzed [12]. A CSSS consisting of one SU source, multiple SU relays, one SU destination, and one PU receiver is considered [13]. Neglecting the presence of the direct link, the outage performance of the secondary network with an appropriate relay selection criterion has been considered. The outage probability of SU in a multi-user and multi-relay spectrum sharing system employing decode-and-forward (DF) and amplify-and-forward (AF) schemes has been derived over Rayleigh fading channels [14]. Two novel automatic-repeat-request (ARQ) based protocols have been proposed in [15], which exploit cooperation opportunity inherent in secondary retransmission to create access opportunities. In [16], considering the interference power constraint, a tight lower bound and an exact outage probability (OP) are derived for decode-and-forward (DF) underlay cognitive relay network. Recently the effects of PU interference on the secondary AF and DF cognitive radio networks have been investigated in [17-19]. In [17-19] authors, considered the effect of a single PU on the performance of SU, our present work considers the impact of multiple PUs. The performance of cognitive relay network with multiple primary users (PUs) and a single relay has been considered in [20-23]. A cognitive opportunistic relay se- 
lection scheme under spectrum-sharing environment in the presence of multiple PUs has been proposed [22]. The outage performance of a dual-hop DF spectrum sharing system with multiple PU receivers and multiple SU destinations over Nakagami- $m$ fading channels has been investigated [23]. The outage performance of a cognitive radio network in absence of relay under the multiple primary users (PUs) and a secondary user (SU) has been investigated [24]. However, the key limitation of the aforementioned works [20-23] is that the effect of PUs interference at the secondary receiver and relays is neglected. Furthermore, no constraints on primary outage and peak transmit power of SU-Tx or SU relays are considered in [20-23].

In this paper, we study the outage performance of a dualhop cooperative spectrum sharing system (CSSS) under the joint constraints of the primary user outage probability and the peak transmit power of the secondary user. The CSSS model consists of one secondary source, multiple SU relays, one SU destination, and multiple PUs. In a practical network, the secondary receiver (SU-Rx) may receive interference from multiple PUs which limit the performance of SU network. In our model, the SU transmission interferes with the primary users, and the primary users transmission also interferes with the secondary receiver and relays. This is in contrast to earlier work reported in [20-23], where the effect of PUs interference at the secondary receiver and relays is neglected. Furthermore, constraints on primary outage and peak transmit power of SU-Tx or SU relays were not considered simultaneously for multiple PU in early works which are incorporated in our present work. The joint outage constraint of the PU and peak transmit power constraint of the SU on the outage performance of a cognitive cooperative radio network (CCRN) using the proactive and reactive DF schemes has been investigated in [10] for a single PU. In contrast to [10], our present work considers the impact of multiple PUs. In a realistic scenario more than one PU band may be available. Some of them could be unused while others may be occupied depending on PU activity. This feature is captured in our present work while a scenario with a single PU only has been considered in [10]. Moreover, novel closed form expressions for the outage of secondary user in proactive and reactive relay schemes under multiple PUs are derived. The analytical expressions are also supported by simulation in MATLAB.

More precisely the main contributions of our present work can be described as follows: (i) The outage performance of a cognitive radio network with a pair of secondary transmitter and receiver is investigated in the presence of multiple primary users and multiple secondary relays considering the interference from PU. (ii) An adaptive power allocation for secondary transmitter and secondary relays is formulated under the joint outage constraints of the primary user outage and peak transmit power of the secondary users. (iii) Closed from analytical expressions on outage of secondary user are developed for both the proactive and reactive relay schemes following our adaptive power allocation in presence of multiple PUs. (iv) We have analyzed the effects of multiple primary users, based on assumption of a realistic model, where all the primary users are not active at the same time/location and compared our model with a single primary user.

The remainder of this paper is structured as follows. In Sec. 2, the network and channel model are introduced. In Sec. 3, the adaptive transmit power strategy under the outage constraint of multiple PUs is investigated. On this basis, the outage probability of the secondary system is derived. In Sec. 4, numerical results are presented and discussed. Finally, conclusions and future research are presented in Sec. 5.

\section{System and Channel Model}

\subsection{CCRN and Channel Model}

Let us consider a CCRN where the secondary transmitter (SU-Tx) communicates with a secondary receiver (SU$\mathrm{Rx}$ ) through the help of $K$ secondary relay (SRs) who work in DF mode as shown in Fig. 1. The secondary network utilizes the licensed frequency bands allocated to a number of PUs for their communication, i.e., the secondary user is using the entire available band width of the total number of primary users in the system. In particular, the SUs use $M$ licensed frequency bands each of which has the same bandwidth, $B$. It is also assumed that only $N$ out of $M$ frequency bands, $1 \leq N \leq M$, are occupied by $N$ pairs of PUs. It should be noted that the SUs can simultaneously access frequency bands with the PUs as long as the negative effect to the QoS of the PUs due to transmission of SU is controlled. The transmit power of SU-Tx must be controlled so as not to exceed the interference threshold as tolerated by the PUs. Here, we assume that no direct link, between SU-Tx and SURx exists due to severe shadowing. The channel coefficients experience Rayleigh fading and all noise terms are additive white Gaussian noise (AWGN) signals with mean power $N_{0}$. The channel gains of $\mathrm{SU}-\mathrm{Tx} \rightarrow \mathrm{SR}_{k}, \mathrm{SR}_{k} \rightarrow \mathrm{SU}-\mathrm{Rx}$ and $\mathrm{PU}-\mathrm{Tx}_{n} \rightarrow \mathrm{PU}-\mathrm{Rx}_{n}$ communication links are denoted by $g_{k}, h_{k}$ and $\alpha_{n}$ respectively. Furthermore, the channel gains of the $\mathrm{SU}-\mathrm{Tx} \rightarrow \mathrm{PU}-\mathrm{Rx}_{n}, \mathrm{SR}_{k} \rightarrow \mathrm{PU}-\mathrm{Rx}_{n}$, $\mathrm{PU}-\mathrm{Tx}_{n} \rightarrow \mathrm{SR}_{k}$ and $\mathrm{PU}-\mathrm{Tx}_{n} \rightarrow \mathrm{SU}-\mathrm{Rx}$ interference links are denoted by $g_{0, n}, \beta_{n, k}, f_{n, k}$ and $f_{0, n}$ respectively, where $n=\{1,2, \ldots, N\}$ and $k=\{1,2, \ldots, K\}$. We assume that all channel gains are independent and identically distributed exponentially random variables (RVs). Such assumptions are widely used in the literatures [10], [14], [16], [17], [24] which make the problem mathematically tractable. Accordingly, the channel mean powers of $g_{k}, h_{k}, \alpha_{n}, g_{0, n}$, $\beta_{n, k}, f_{n, k}$ and $f_{0, n}$ are denoted by $\sigma_{g}, \sigma_{h}, \sigma_{\alpha}, \sigma_{g, 0}, \sigma_{\beta}$, $\sigma_{f}$, and $\sigma_{f, 0}$ respectively. Perfect channel state informations between SU-Tx and PU-Rx as well as SRs and PU-Rx are assumed to be available at the SU-Tx and SRs respectively. Communication in the secondary network is assumed to occur in a time slotted manner which is described below. 


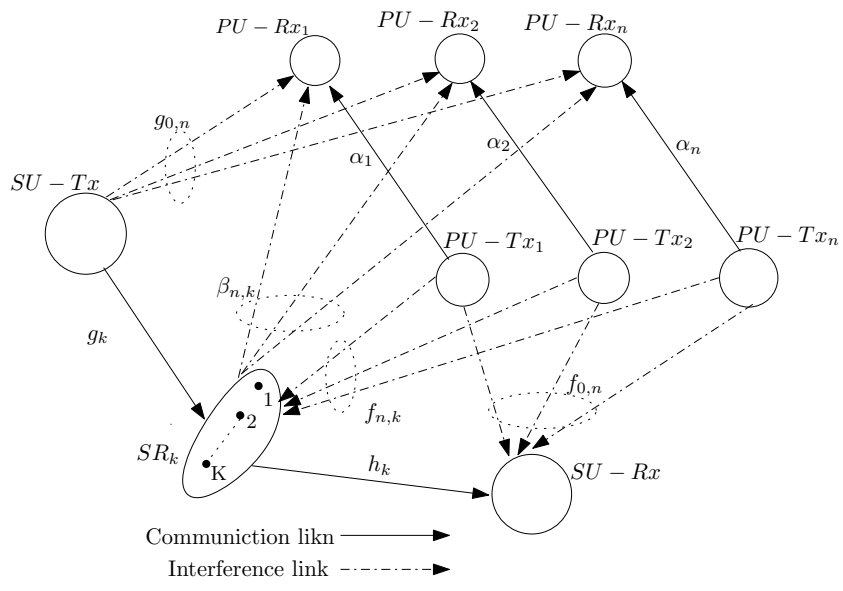

Fig. 1. System model of a cognitive cooperative radio network.

In the first time slot, the SU-Tx broadcasts its signals to $K \mathrm{SRs}$ and the signal-to-interference plus noise ratio (SINR) at secondary relay $\mathrm{SR}_{k}$ is expressed as [24]

$$
\gamma_{\mathrm{SR}_{k}}=\frac{P_{s} g_{k}}{\sum_{n=1}^{N} P_{n} f_{n, k}+B_{s} N_{0}}
$$

where $P_{n}, P_{s}$ and $N_{0}$ are PU average transmit power of the $\mathrm{PU}-\mathrm{Tx}_{n}, \mathrm{SU}-\mathrm{Tx}$ instantaneous transmit power and noise power respectively. In order to guarantee that no PU is subjected to harmful interference due to transmission of SU, the transmit power of the SU-Tx should be controlled so as to satisfy the outage constraint of the PU under the worst SINR as follows [24]

$$
\begin{aligned}
& P_{\text {Out }}^{P_{1}}=\operatorname{Pr}\left[\min _{n=1,2, \ldots, N}\left(\frac{P_{n} \alpha_{n}}{P_{s} g_{0, n}+B N_{0}}\right)<\gamma_{\text {th }}^{P}\right]<\varepsilon \\
& =1-\prod_{n=1}^{N}\left[1-\left\{\operatorname{Pr}\left(\frac{P_{n} \alpha_{n}}{P_{s} g_{0, n}+B N_{0}}<\gamma_{\text {th }}^{P}\right)\right]<\varepsilon,\right.
\end{aligned}
$$

under the constraint of

$$
P_{s} \leq P_{p k_{1}}
$$

where $\gamma_{\mathrm{th}}^{p}=2^{\frac{r_{p}}{B}}-1, p$ and $\varepsilon$ denote the SINR threshold of PU, outage transmission rate for the PUs and outage constraint of the PU-Rx respectively, while $P_{p k_{1}}$ stands for the SU-Tx peak transmit power. Assuming that the PU-Txs transmit powers are fixed and identical, i.e., $P_{n}=P_{p}$ for $n=1,2, \ldots, N$.

In the second time slot, one relay is selected, based on any one of proactive or reactive protocol say $\mathrm{SR}_{k}$, to forward the signal to the SU-Rx. The SINR at the SU-Rx is now expressed as:

$$
\gamma_{\mathrm{R}_{k} D}=\frac{P_{\mathrm{R}_{k}} h_{k}}{\sum_{n=1}^{N} P_{p} f_{0, n}+B_{s} N_{0}} .
$$

As in the first time slot, the $S R_{k}$ also adjusts its transmit power to guarantee the outage constraint of the PUs and the limitation on peak transmit power of the $S R_{k}$, i.e.,

$$
P_{\text {Out }}^{P_{2}}=\operatorname{Pr}\left[\min _{n=1,2, \ldots, N}\left(\frac{P_{n} \alpha_{n}}{P_{R_{k}} \beta_{n, k}+B N_{0}}\right)<\gamma_{\text {th }}^{P}\right]<\varepsilon,
$$

$$
P_{\mathrm{R}_{k}} \leq P_{p k_{2}}
$$

where $P_{p k_{1}}$ denotes the peak transmit power of $k$ 'th secondary relay $\mathrm{SR}_{k}$.

\subsection{Proactive and Reactive DF Schemes}

In order to enhance the system performance, the potential SR can be selected following the proactive or reactive DF schemes as in [10]. Specifically, in the proactive DF scheme, the best SR is chosen prior to the SU-Tx transmission to maximize the minimum of the SINR between the SU $-\mathrm{Tx} \rightarrow \mathrm{SR}_{k}$ and $\mathrm{SR}_{k} \rightarrow \mathrm{SU}-\mathrm{Rx}$ links for $k=1,2, \ldots, K$. Thus, the end-to-end SINR is given by [10]

$$
\gamma^{\text {Pro }}=\max _{k \in\{1,2, \ldots, K\}}\left[\min \left\{\gamma_{\mathrm{SR}_{k}}, \gamma_{\mathrm{R}_{k} D}\right\}\right]
$$

In the reactive DF scheme, only the SRs that successfully decode the message in the first hop regenerate and transmit it in the second hop. Thus, the transmissions in the second hop are performed only by a subset $\xi$ of $N$ SRs, given as [10]

$$
\xi=\left\{k \in(1,2, \ldots, K) ; \gamma_{\mathrm{SR}_{k}} \geq \mu_{\mathrm{th}}\right\}
$$

where $\xi$ is a set of $k_{1}$ SRs which are able to decode successfully the received message from the SU-Tx. In Eq. 8, the decoding process at $\mathrm{SR}_{k}$ is successful if, $\left\{\gamma_{\mathrm{SR}_{k}} \geq \mu_{\text {th }}\right\}$, i.e., there is no outage event in the first hop. Next based on the decodable set $\xi$, one SR is chosen to maximize the instantaneous SINR between the $\mathrm{SR}_{k} \rightarrow \mathrm{SU}-\mathrm{Rx}$ links for all $k \in \xi$ is given as [10]

$$
\gamma^{\mathrm{Re}}=\max _{k \in \xi}\left\{\gamma_{\mathrm{R}_{k} D}\right\}
$$

\section{Performance Analysis}

To investigate the power allocation policy for SU - Tx and $\mathrm{SR}_{k}$, the following property is used.

Property 1: Consider $X, Y_{i}(i=1,2, \ldots, P)$ and $Z$ are random variables $(\mathrm{RVs})$, where $Z$ is defined as:

$$
Z=\frac{a X}{\sum_{i=1}^{P} b Y_{i}+c} \quad \text { with } \quad b, c, P \geq 0 .
$$

The RVs $X$ and $Y_{i}(i=1,2, \ldots, P)$ are exponentially distributed with mean $\sigma_{x}$ and $\sigma_{y_{i}}$, respectively. For the convenience of theoretical analysis, consider mean of $Y_{i}$ $(i=1,2, \ldots, P)$ are equal, i.e., $\sigma_{y_{i}}(i=1,2, \ldots, P)=\sigma_{y}$. The cumulative distribution function (CDF) of random variable $Z$ is given as [24]

$F_{z}\left(a, b, c, P, \sigma_{x}, \sigma_{y}, z\right)=1-\left(1+\frac{b z \sigma_{y}}{a \sigma_{x}}\right)^{-P} \exp \left(-\frac{z c}{a \sigma_{x}}\right)$

Proof: The proof can be found in [24]. 


\subsection{Power Allocation Policy for the SU-Tx}

Applying Property 1 to the term of outage probability, $P_{\text {Out }}^{p_{1}}$, given in (2), we have

$P_{\text {Out }}^{P_{1}}=1-\left[\left(1+\frac{P_{s} \sigma_{g 0} \gamma_{\mathrm{th}}^{p}}{P_{p} \sigma_{\alpha}}\right)^{-1} \exp \left(-\frac{\gamma_{\mathrm{th}}^{p} B N_{0}}{\sigma_{\alpha} P_{p}}\right)\right]^{N} \leq \varepsilon$.

We assumed fading in different wireless channel are independent and identically and substituting (12) into (2), and after some manipulations, the maximal transmit power of the SU-Tx under the outage constraints of all the PUs is found as

$$
P_{s} \leq \frac{P_{p} \sigma_{\alpha}}{\gamma_{\mathrm{th}}^{p} \sigma_{g, 0}} \psi
$$

where $\psi=\max \left(0, \frac{1}{\{1-\epsilon\}^{\frac{1}{N}}} \exp \left\{-\frac{\gamma_{\mathrm{th}}^{p} B N_{0}}{\sigma_{\alpha} P_{p}}\right\}-1\right)$. The expression (13) holds for the assumptions of iid fading in all the links. Combining (13) with (3), an adaptive power allocation policy for SU-Tx is established as [10]

$$
\varphi_{s}=\min \left[P_{p k_{1}}, \frac{P_{p} \sigma_{\alpha}}{\gamma_{\mathrm{th}}^{p} \sigma_{g, 0}} \psi\right]
$$

\subsection{Power Allocation Policy of the Secondary Relay SR}

In the second time slot, the received signal is decoded by relays using proactive or reactive DF scheme. The decoded signal is then transmitted by the selected relays to the secondary receiver. The relays should adjust their transmit power to satisfy its peak transmit power and the outage constraint of the primary network. By using the same result as in section 3.1, the outage probability of the PU in worst condition under the effect of interference from secondary relays is written as

$$
P_{\text {Out }}^{P_{2}}=1-\left[\left(1+\frac{P_{\mathrm{R}_{k}} \sigma_{\beta} \gamma_{\mathrm{th}}^{p}}{P_{p} \sigma_{\alpha}}\right)^{-1} \exp \left(-\frac{\gamma_{\mathrm{th}}^{p} B N_{0}}{\sigma_{\alpha} P_{p}}\right)\right]^{N} \leq \varepsilon
$$

and the adaptive transmit power allocation policy for the $\mathrm{SR}_{k}$ is given by [10]

$$
\varphi_{\mathrm{R}_{k}}=\min \left[P_{p k_{2}}, \frac{P_{p} \sigma_{\alpha}}{\gamma_{\mathrm{th}}^{p} \sigma_{\beta}} \psi\right]
$$

It is assumed that the channel mean powers of the $\mathrm{SU}-\mathrm{R}_{k} \rightarrow \mathrm{PU}-\mathrm{Rx}$ links $\sigma_{\beta}$ are identical for all relays. Therefore, $\varphi_{\mathrm{R}_{k}}$ given in (16) are identical and can be written as $\varphi_{\mathrm{R}_{k}}=\varphi_{\mathrm{R}}$ for $\{\forall k \in 1,2, \ldots, K\}$.

\subsection{Outage Probability for the Proactive DF Scheme}

The outage probability is defined as the probability that the end-to-end SINR of the secondary network is dropped be- low a given threshold. By using (7), (14) and (16), the outage probability for the proactive DF scheme can be expressed as

$$
\begin{array}{r}
P_{\text {Out }}^{\text {Pro }}=\operatorname{Pr}\left(\gamma^{\text {Pro }}<\gamma_{\mathrm{th}}^{s}\right) \\
\int_{0}^{\infty} \prod_{k=1}^{K}\{1-\underbrace{\operatorname{Pr}\left(\frac{\varphi_{s} g_{k}}{\sum_{n=1}^{N} P_{p} f_{n, k}+B_{s} N_{0}}>\gamma_{\mathrm{th}}^{s}\right)}_{I} \\
\underbrace{\operatorname{Pr}\left(\frac{\varphi_{R_{k}} h_{k}}{P_{p} x+B_{s} N_{0}}>\gamma_{\mathrm{th}}^{s}\right)}_{J}\} f_{X}(x) \mathrm{d} x .
\end{array}
$$

The term $I$ is derived by applying Property 1 as

$$
I=\left(1+\frac{P_{p} \sigma_{f} \gamma_{\mathrm{th}}^{s}}{\varphi_{s} \sigma_{g}}\right)^{-N} \exp \left(-\frac{\gamma_{\mathrm{th}}^{s} B_{s} N_{0}}{\sigma_{g} \varphi_{s}}\right)
$$

where $\gamma_{\mathrm{th}}^{s}=2^{\frac{r_{s}}{B_{s}}}-1$ is the outage threshold of the CCRN. The term $J$ in (17) can be expressed as

$$
J=\exp \left(-\frac{\gamma_{\mathrm{th}}^{s}\left\{P_{p} x+B_{s} N_{0}\right\}}{\varphi_{R_{k}} \sigma_{h}}\right)
$$

where $X=\sum_{n=1}^{N} f_{0, n}$ are gamma distributed RVs and PDF of RVs $X$ can be written as

$$
f_{X}(x)=\frac{x^{N-1}}{\sigma_{f 0}^{N} \Gamma(N)} \exp \left(-\frac{x}{\sigma_{f 0}}\right) .
$$

Substituting equations (18), (19) and (20) into (17), the outage probability for the proactive DF scheme is expressed as

$$
\begin{aligned}
& P_{\text {Out }}^{\text {Pro }}=\frac{1}{\sigma_{f 0}^{N}} \int_{0}^{\infty}\left[1-I \exp \left(-\frac{\gamma_{\mathrm{th}}^{s}\left\{P_{p} x+B_{s} N_{0}\right\}}{\varphi_{\mathrm{R}_{k}} \sigma_{h}}\right)\right]^{K} \\
& \frac{x^{N-1}}{\Gamma(N)} \exp \left(-\frac{x}{\sigma_{f 0}}\right) \mathrm{d} x \\
& =\frac{1}{\sigma_{f 0}^{N}} \sum_{t}^{K} C_{t}^{K}(-I)^{t} \exp \left(-\frac{t \gamma_{\mathrm{th}}^{s} B_{s} N_{0}}{\varphi_{\mathrm{R}_{k}} \sigma_{h}}\right) \\
& \left(\frac{1}{\sigma_{f 0}}+\frac{t \gamma_{\mathrm{th}}^{s} P_{p}}{\varphi_{\mathrm{R}_{k}} \sigma_{h}}\right)^{-N}
\end{aligned}
$$

where $C_{a}^{b}$ is a binomial coefficient defined by $C_{a}^{b}=\frac{b !}{a !(b-a) !}$.

\subsection{Outage Probability for the Reactive DF Scheme}

According to the law of total probability, the outage probability of the Reactive DF scheme can be formulated as [10]

$$
P_{\text {Out }}^{\mathrm{Re}}=\sum_{k_{1}=0}^{K} C_{k_{1}}^{K} \operatorname{Pr}\left[\gamma^{\mathrm{Re}}<\gamma_{\mathrm{th}}^{s} \mid \xi\right] \operatorname{Pr}\{\xi\} .
$$

In the Reactive DF scheme only a subset $\xi$ of $K$ secondary relays perform the transmission. This subset is the set of $k_{1}$ 
relay out of $K$ who are able to decode the transmission of SU successfully. The probability of this transmission is given by $\operatorname{Pr}\{\xi\}$. This probability is written as

$$
\operatorname{Pr}\{\xi\}=\prod_{i \in \xi} \operatorname{Pr}\left\{\gamma_{\mathrm{SR}_{i}}>\mu_{\mathrm{th}}\right\} \prod_{i \notin \xi} \operatorname{Pr}\left\{\gamma_{\mathrm{SR}_{i}} \leq \mu_{\mathrm{th}}\right\} .
$$

The two factors in (23) can be calculated by using Property 1 and $\operatorname{Pr}\{\xi\}$ can be written as

$$
\operatorname{Pr}\{\xi\}=[1-A]^{k_{1}}[A]^{K-k_{1}}
$$

where $A=F_{z}\left(\varphi_{s}, P_{p}, B_{s} N_{0}, N, \sigma_{g}, \sigma_{f}, \alpha_{\text {th }}\right)$. Additionally as the events $\operatorname{Pr}\left\{\gamma^{\operatorname{Re}}<\gamma_{\text {th }}^{s}\right\}$ and $\operatorname{Pr}\{\xi\}$ are independent, the term $\operatorname{Pr}\left\{\gamma^{\operatorname{Re}}<\gamma_{\text {th }}^{s} \mid \xi\right\}$ in (22) can be written as

$$
\begin{array}{r}
\operatorname{Pr}\left\{\gamma^{\mathrm{Re}} \leq \gamma_{\mathrm{th}}^{s} \mid \xi\right\}=\operatorname{Pr}\left[\max _{k \in \xi}\left\{\gamma_{\mathrm{R}_{k} D}\right\} \leq \gamma_{\mathrm{th}}^{s}\right] \\
=\int_{0}^{\infty} \operatorname{Pr}\left[\max _{k \in \xi}\left(\frac{\varphi_{\mathrm{R}_{k}} h_{k}}{P_{p} x+B_{s} N_{0}}\right) \leq \gamma_{\mathrm{th}}^{s}\right] f_{X}(x) \mathrm{d} x \\
=\frac{1}{\sigma_{f 0}^{N}} \int_{0}^{\infty}\left[1-\exp \left(-\frac{\gamma_{\mathrm{th}}^{s}\left\{P_{p} x+B_{s} N_{0}\right\}}{\varphi_{\mathrm{R}_{k}} \sigma_{h}}\right)\right]^{k_{1}} \\
\times \frac{x^{N-1}}{\Gamma(N)} \exp \left(-\frac{x}{\sigma_{f 0}}\right) \mathrm{d} x \\
=\frac{1}{\sigma_{f 0}^{N}} \sum_{t=0}^{k_{1}} C_{t}^{k_{1}}(-1)^{t} \exp \left(-\frac{t \gamma_{\mathrm{th}}^{s} B_{s} N_{0}}{\varphi_{\mathrm{R}_{k}} \sigma_{h}}\right) \\
\left(\frac{1}{\sigma_{f 0}}+\frac{t \gamma_{\mathrm{th}}^{s} P_{p}}{\varphi_{\mathrm{R}_{k}} \sigma_{h}}\right)^{-N} .
\end{array}
$$

Finally, substituting equations (24) and (25) into (22), the outage probability for the reactive DF scheme can be written as in (26)

$$
\begin{aligned}
P_{\text {Out }}^{\mathrm{Re}}= & \sum_{k_{1}=0}^{K} \sum_{t=0}^{k_{1}} \frac{C_{k_{1}}^{K} C_{t}^{k_{1}} A^{K-k_{1}}(1-A)^{k_{1}}(-1)^{t}}{\sigma_{f 0}^{N}} \\
& \exp \left(-\frac{t \gamma_{\mathrm{th}}^{s} B_{s} N_{0}}{\varphi_{\mathrm{R}^{*}} \sigma_{h}}\right)\left(\frac{1}{\sigma_{f 0}}+\frac{t \gamma_{\mathrm{th}}^{s} P_{p}}{\varphi_{R_{k}} \sigma_{h}}\right)^{-N} .
\end{aligned}
$$

It may be noted that the (21) and (26) on outage probability of $\mathrm{SU}$ for proactive and reactive case respectively are our novel development.

\section{Results and Discussion}

In this section, numerical results are provided to illustrate the impact of primary networks on the cognitive radio systems performance. Results based on our analytical formulation in previous section are provided. Furthermore, MATLAB based simulation is carried out and results based on simulation are found to match the analytical results. Note that in all the figures in this section, analytical results are represented by continuous curves, while the simulation results are represented by discrete marks on the curves. The following arbitrary parameters are chosen to evaluate the performance of the network under present study which is in line

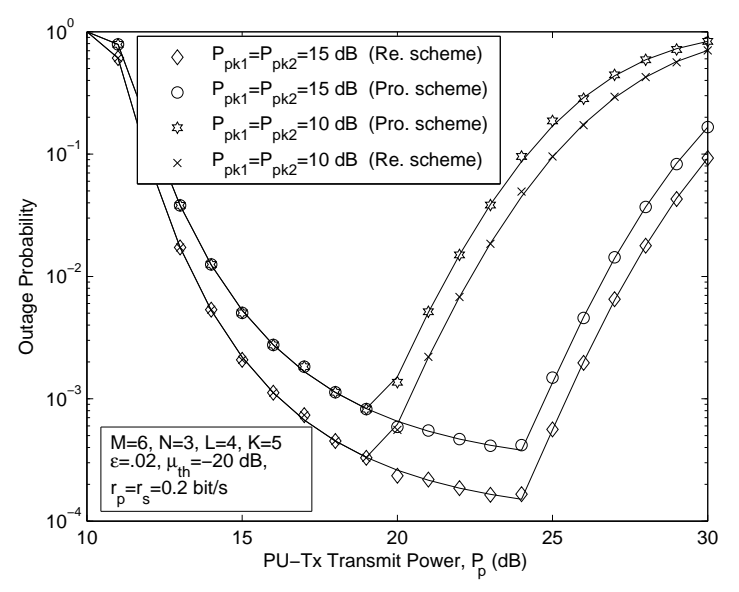

Fig. 2. Outage probability of SU versus PU-Tx transmit power $P_{p}$ with different peak transmit powers $P_{p k_{1}}=P_{p k_{2}}=$ $10,15 \mathrm{~dB}$.

with [10], [24]. Different values of peak transmit power of SU-Tx and SRs, i.e., $P_{k_{1}}=P_{k_{2}}=10,15 \mathrm{~dB}$, outage transmission rate for the SU and SRs equal, i.e., $r_{s}=r_{p}=0.2 \mathrm{bit} / \mathrm{s}$, outage constraint of the PUs $\epsilon=0.02$ and bandwidth $B$ and $N_{0}$ are nominalised to 1 .

Figure 2 plots the outage probability of the secondary network for several values of peak transmit power $P_{p k_{1}}=$ $P_{p k_{2}}=10,15 \mathrm{~dB}$, the number of active PUs $N=3$, the number of licensed frequency bands $M=6$ and the number of secondary relays $K=5$. Moreover, it is interesting to observe that the outage probability of both the schemes reduces with increase in PU-Tx power until $P_{p}$ is smaller than an optimal value $\left(P_{p} \leq 15,19 \mathrm{~dB}\right.$ respectively) where it is minimum at the said optimal value. Beyond this optimal value (i.e., $P_{p}=15$ and $19 \mathrm{~dB}$ under two different cases) outage probability increase gradually. This is in tune with behaviour observed in [10] for the case of a single PU. Increasing $P_{p}$ leads to an increase in transmission rate of the primary user, i.e., improvement in the performance of the primary network. Furthermore, as $P_{p}$ continuously increases, the transmit power of SU and SR, i.e., $\varphi_{s}$ and $\varphi_{\mathrm{R}_{k}}$ increase following (14) and (16), i.e., the performance of the secondary network improves. However, if the PU-Tx transmit power $P_{p}$ is increased beyond the optimal value, $\varphi_{s}$ and $\varphi_{\mathrm{R}_{k}}$ cannot be further increased due to the peak transmit power constraint.

Figure 3 shows outage probability of the proactive and reactive DF schemes versus PU-Tx transmit power $P_{p}$ for several values of outage threshold in the first hop $\mu_{t h}=-5,-16,-20 \mathrm{~dB}$. It can be clearly seen that the outage performance of the reactive scheme outperforms the proactive scheme at $\mu_{\mathrm{th}}=-20 \mathrm{~dB}$ and overlaps with that of the proactive scheme as $\mu_{\mathrm{th}}=-16 \mathrm{~dB}$. This is due to the fact that the number of SRs in the decoding set of the reactive scheme is increased as the outage threshold in the first hop decreases. Accordingly, the relay selection, in the second hop, is more diverse. As a result, the outage performance of the reactive scheme is improved. 


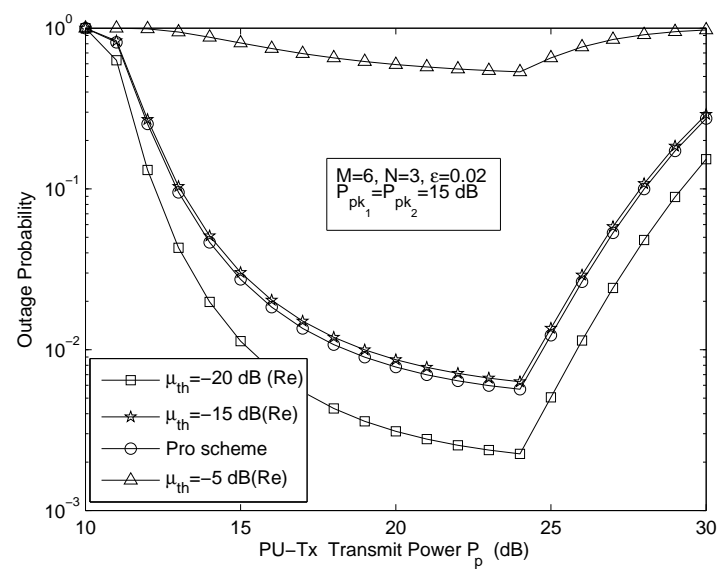

Fig. 3. Outage probability of SU with proactive and reactive DF schemes for different values of outage threshold in the first hop.

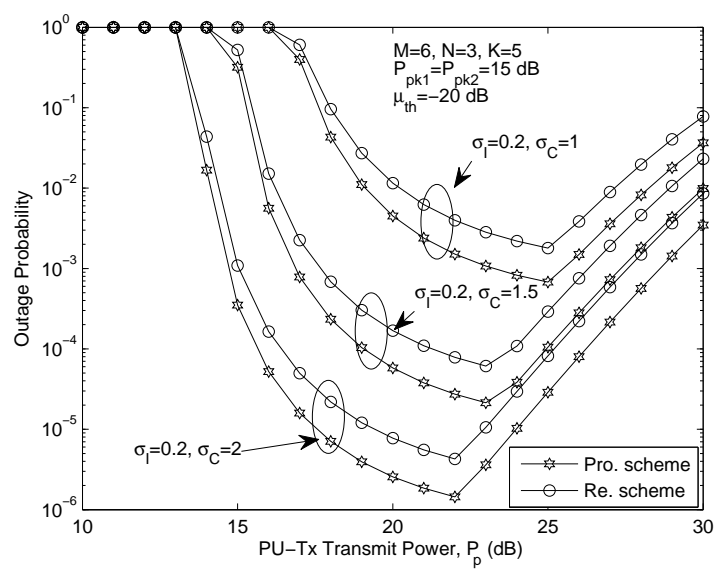

Fig. 4. Outage probability of the secondary network with different values of channel mean power of the communication link $\sigma_{C}=1,1.5,2$.

All communication and interference links are considered to be independent and identically distributed Rayleigh fading having equal channel mean power $\sigma_{C}$ and $\sigma_{I}$ respectively. Figure 4 plots the outage probability of the secondary network for several values of $\sigma_{C}=1,1.5,2$ while $\sigma_{I}$ is fixed at 0.2. The number of active PUs $N=3$, the number of licensed frequency bands $M=6$ and number of relays $K=5$. The outage probability reduces as the channel mean power $\sigma_{C}$ increases, i.e., the channel condition of the PUs communication links is improved. Primary user may reduce its transmit power which reduces the interference on SU. As a result, the outage performance of the secondary network is improved. Additionally, we also observe that the outage probability decreases as $P_{p}$ increases to a specific value, e.g., $22 \mathrm{~dB}$, and it increases rapidly as $P_{p}$ exceeds $22 \mathrm{~dB}$ with $\sigma_{C}=2$ due to the same reason as explained in connection with Fig. 2.

Figure 5 shows the impact of channel mean powers of the interference links, i.e., several values of $\sigma_{I}=0.7,0.5$ and 0.3 are assumed while $\sigma_{C}$ is kept fixed at 2. As expected, when the channel mean powers of the interference

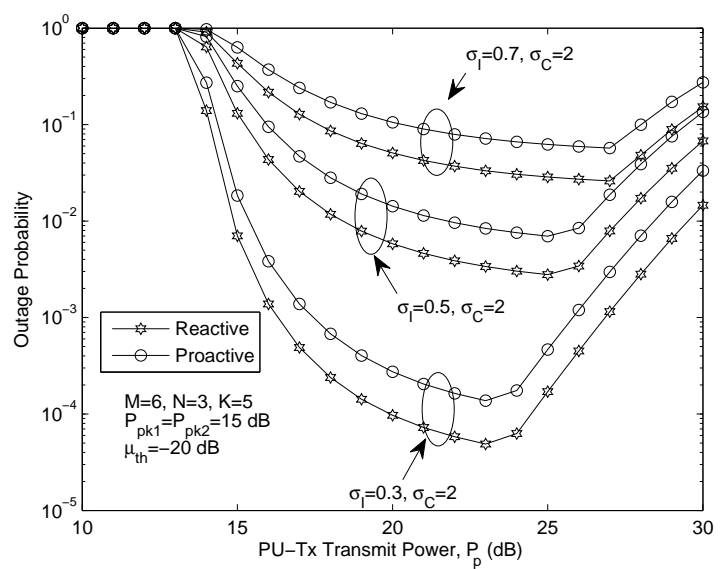

Fig. 5. Outage probability of the secondary network with different values of channel mean power of the interference link $\sigma_{I}=0.7,0.5,0.3$.

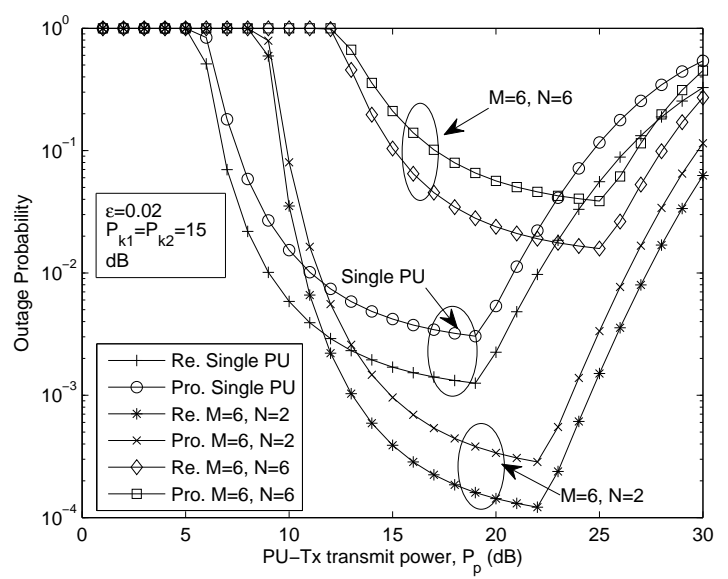

Fig. 6. Outage probability of the secondary network with different number of active PUs $N=2$ to 6 on the licensed frequency bands.

links increases, the outage probability of SU increases due to increased interference from PU.

Figure 6 represents the effects of available as well as active primary users on outage performance of secondary user. It is seen that the secondary user performance degrade as the number of active primary users increases from 2 to 6 . This is due to the fact that the SU-Tx must control its transmit power to satisfy the constraint corresponding to worst case of SU-Tx to PU-Rx link, which imposes a limit on transmit power of secondary user. The outage performance of SU for the case of a single PU is also shown. It may be noted that the result for a single PU case using our formulation matches with that of [10]. It is observed from the Fig. 6 that the performance of SU emproves for multiple PU case where all the PUs are not active. This observation is important as in a practical model, 15 to $85 \%$ of the primary users remain idle at any given time/location [25]. The optimal transmit power $P_{p}$ depends on various parameters such as peak transmit power of SU-Tx and SRs $\left(P_{p k_{1}}, P_{p k_{2}}\right)$, channel links $\left(\sigma_{I}\right.$ and $\sigma_{C}$ ) and number of active PUs $(N)$. It is seen that as the number of active PUs increases, the switching from low 


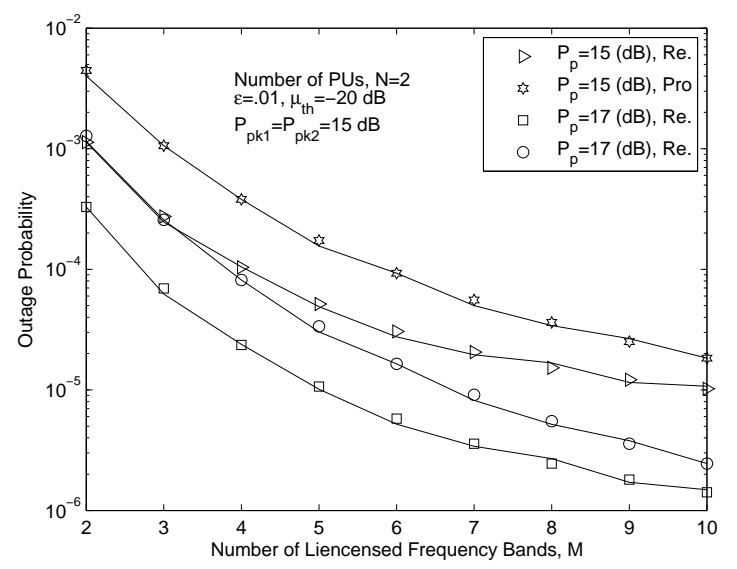

Fig. 7. Outage probability of the secondary network as a function of the number of licensed frequency bands with $P_{p k_{1}}=P_{p k_{2}}=15 \mathrm{~dB}$ and $\mu_{t h}=-20 \mathrm{~dB}$.

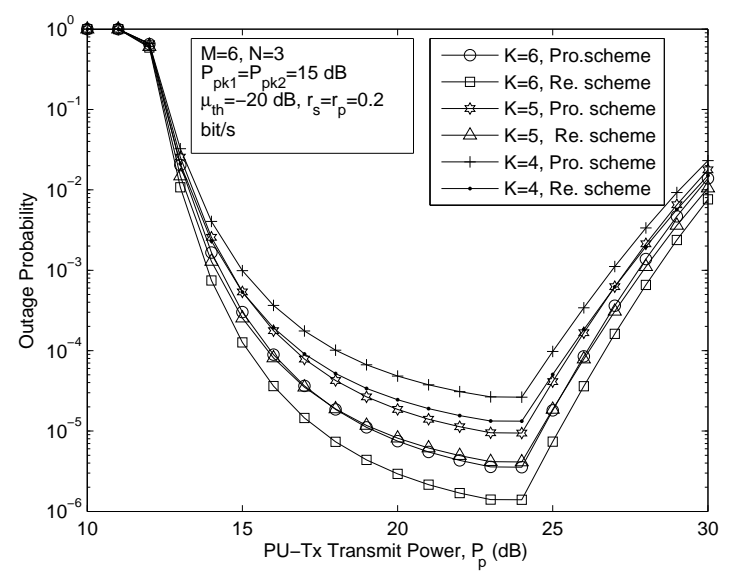

Fig. 8. Impact of number of cognitive relay (K) on outage of CCRN.

to high outage occurs at higher PU-Tx transmit power $P_{p}$. This behaviour can be explained as follows: In the case $M$ is fixed, i.e., $B_{s}=M B$ is fixed and as number of active PU $(N)$ increases to $M$, then $\psi$ in (14) and (16) decreases as it is reciprocally related to $N$, having the relationship of $\frac{1}{(1-\xi)^{\frac{1}{N}}}$. Under such situation, it may be possible to increase the PU power $\left(P_{p}\right)$ while maintaining the SU-Tx power with in its constraint as per power allocation policy in (14).

Figure 7 shows the outage probability as a function of the number of licensed frequency bands for different values of PU transmit powers, $P_{p}=15,17 \mathrm{~dB}$ and the number of active PUs being $N=2$. We observe from the Fig. 7 that the outage probability increases rapidly as the PU-Tx transmit power $P_{p}$ increases. Moreover, the outage probability decreases significantly as the number of radio frequency bands increases, i.e., the bandwidth available to the SU increases. Clearly, the performance of the secondary network can be improved by increasing the bandwidth. However, if the number of active PUs increases, the performance of the secondary network is degraded.

In Fig. 8, we investigate the effects of the number of secondary relays on the outage performance for both the proac- tive and reactive schemes respectively. In this scenario, the number of active PUs $N=3$ and the number of licensed frequency bands $M=6$, while the number of relays $K$ is varied from 4 to 6 . As expected, the outage performance improves with increase in the number of relays, which confirms (21) and (26) for proactive and reactive DF schemes respectively. This occurs because the diversity order improves when the number of relays increases. As we increase the number of cognitive relay from $K=4$ to 6 , at a PU-Tx transmit power $\left(P_{p}\right)$ of $24 \mathrm{~dB}$, the outage probability of secondary user is increased by almost $90 \%$ in both the schemes.

\section{Conclusion}

In this paper, we have studied the performance of proactive and reactive DF relaying schemes in terms of outage probability in CCRN under the joint outage constraint of multiple PUs and peak transmit power constraint of the SU-Tx and SRs. An adaptive power control policy for the secondary transmitter and secondary relay is derived considering proactive and reactive relay protocols. A closed-form expression for the outage probability has been derived for the secondary system(i.e., SU-Tx and SRs). It is seen that the outage probability of secondary system improves with increase in the number of cognitive relays in a multi-relay cooperative spectrum sharing schemes. The outage probability which deteriorates with higher interference from multiple PUs can be compensated by increasing the number of relays. It is seen that outage of SU reaches a minimum value for a transmit power PU, depending on the number of relays, number of PUs and other network parameters. More importantly, our results indicates that the performance of the CCRN can be improved by increasing the bandwidth over a number of licensed frequency bands. However, the performance of the CCRN degrade significantly if the number of active PUs increases. Moreover, in reality, the secondary outage performance will improve using bands of multiple PUs (when all PUs are not simultaneously active) as compared to a single primary user. The above study is helpful in designing and assessing the performance of a relay based secondary system coexisting with multiple PUs across multiband.

\section{References}

[1] MITOLA, J., MAGUIRE, G. Q. Cognitive radio: making software radios more personal. IEEE Personal Communications, 1999, vol. 6, no. 4, p. 13-18. DOI: $10.1109 / 98.788210$

[2] GHASEMI, A., SOUSA, E. Fundamental limits of spectrumsharing in fading environments. IEEE Transactions on Wireless Communications, 2007, vol. 6, no. 2, p. 649-658. DOI: 10.1109/TWC.2007.05447

[3] LANEMAN, J., TSE, D., WORNELL, G. W. Cooperative diversity in wireless networks: Efficient protocols and outage behavior. IEEE Transactions on Information Theory, 2004, vol. 50, no. 12, p. 30623080. DOI: 10.1109/TIT.2004.838089 
[4] DE, C. K., KUNDU, S. Adaptive decode-and-forward protocol-based cooperative spectrum sensing in cognitive radio. Inderscience International Journal of Communication Networks and Distributed Systems, 2015, vol. 14, no. 2, p. 117-133. DOI: 10.1504/IJCNDS.2015.067653

[5] DE, C. K., KUNDU, S. Adaptive decode-and-forward protocol based cooperative spectrum sensing in cognitive radio with interference at the secondary users. Wireless Personal Communication, 2014, vol. 79, no. 2, p. 1417-1434 . DOI: 10.1007/s11277-014-1937-2

[6] DE, C. K., SINHA, R., KUNDU, S. Adaptive decode-and-forward protocol based cooperative spectrum sensing in cognitive radio with interference at the secondary users. In Proceedings of the Annual IEEE India Conference (INDICON). (India), 2014, p. 1-6. DOI: 10.1109/INDICON.2014.7030376

[7] BAO, V. N. Q., DUONG, T., BENEVIDES DA COSTA, D., et al. Cognitive amplify-and-forward relaying with best relay selection in non-identical Rayleigh fading. IEEE Communications Letters, 2013, vol. 17, no. 3, p. 475-478. DOI: 10.1109/LCOMM.2013.011513.122213

[8] DING, H., GE, J., DA COSTA, D., et al. Asymptotic analysis of cooperative diversity systems with relay selection in a spectrum-sharing scenario . IEEE Transactions on Vehicular Technology, 2011, vol. 60, no. 2, p. 457-472. DOI: 10.1109/TVT.2010.2100053

[9] KIM, K. J., DUONG, T., TRAN, X.-N. Performance analysis of cognitive spectrum-sharing single-carrier systems with relay selection. IEEE Transactions on Signal Preprocessing, 2012, vol. 60, no. 12, p. 6435-6449. DOI: 10.1109/TSP.2012.2218242

[10] TRAN, H., ZEPERNICK, H.-J., PHAN, H. Cognitive proactive and reactive DF relaying schemes under joint outage and peak transmit power constraints. IEEE Communications Letters, 2013, vol. 17, no. 8, p. 1548-1551. DOI: 10.1109/LCOMM.2013.062113.130573

[11] FAN, Z., GUO, D., ZHANG, B. Outage probability and power allocation for two-way DF relay networks with relay selection. Radioengineering, 2012, vol. 21, no. 3, p. 795-801. ISSN: 1210-2512

[12] ZHONG, C., RATNARAJAH, T., WONG, K.-K. Outage analysis of decode-and-forward cognitive dual-hop systems with the interference constraint in Nakagami-m fading channels. IEEE Transactions on Vehicular Technology, 2011, vol. 60, no. 6, p. 2875-2879. DOI: 10.1109/TVT.2011.2159256

[13] LEE, J., WANG, H., ANDREWS, J., et al. Outage probability of cognitive relay networks with interference constraints. IEEE Transactions on Wireless Communications, 2010, vol. 10, no. 2, p. 390-395. DOI: 10.1109/TWC.2010.120310.090852

[14] GUIMARAES, F., DA COSTA, D., TSIFTSIS, T., et al. Multiuser and multirelay cognitive radio networks under spectrum-sharing constraints. IEEE Transactions on Vehicular Technology, 2014, vol. 63, no. 1, p. 433-439. DOI: 10.1109/TVT.2013.2275201

[15] ZHANG, Z., WU, Q., WANG, J. ARQ protocols in cognitive decodeand-forward relay networks: Opportunities gain. Radioengineering, 2015, vol. 24, no. 1, p. 296-304. DOI: 10.13164/re.2015.0296

[16] LUO, L., ZHANG, P., ZHANG, G., et al. Outage performance for cognitive relay networks with underlay spectrum sharing. IEEE Communications Letters, 2011, vol. 15, no. 7, p. 710-712. DOI: 10.1109/LCOMM.2011.051011.110426

[17] YANG, P., LUO, L., QIN, J. Outage performance of cognitive relay networks with interference from primary user. IEEE Communications Letters, 2012, vol. 16, no. 10, p. 1695-1698. DOI: 10.1109/LCOMM.2012.081612.121086
[18] YANG, P., ZHANG, Q., QIN, J. Exact outage probability of nth best multicast relay networks with co-channel interference. IEEE Communications Letters, 2013, vol. 2, no. 6, p. 595-598. DOI: 10.1109/WCL.2013.081413.130438

[19] YANG, P., ZHANG, Q., LUO, L., et al. Outage performance of underlay cognitive opportunistic multi-relay networks in the presence of interference from primary user. Wireless Personal Communications, 2014, vol. 74, no. 2, p. 343-358. DOI: 10.1007/s11277-013-1288-4

[20] TRAN, H., DUONG, T., ZEPERNICK, H. Performance analysis of cognitive relay networks under power constraint of multiple primary users. In Proceedings of the IEEE Global Telecommunications Conference (GLOBECOM). (USA), 2011, p. 1-6. DOI: 10.1109/GLOCOM.2011.6133587

[21] DUONG, T., YEOH, P. L., BAO, V. N. Q., et al. Cognitive relay networks with multiple primary transceivers under spectrum-sharing. IEEE Signal Processing Letters, 2012, vol. 19, no. 11, p. 741-744. DOI: 10.1109/LSP.2012.2217327

[22] DUONG, T., KIM, K. J., ZEPERNICK, H.-J., et al. Opportunistic relaying for cognitive network with multiple primary users over Nakagami-m fading. In Proceedings of the IEEE International Conference on Communications (ICC). 2013, p. 5668-5673. DOI: $10.1109 / \mathrm{ICC} .2013 .6655497$

[23] BENEVIDES DA COSTA, D., ELKASHLAN, M., YEOH, P. L., et al. Dual-hop cooperative spectrum sharing systems with multiprimary users and multi-secondary destinations over Nakagami-m fading. In Proceedings of the 23rd IEEE International Symposium on Personal Indoor and Mobile Radio Communications (PIMRC). 2012, p. 1577-1581. DOI: 10.1109/PIMRC.2012.6362599

[24] TRAN, H., ZEPERNICK, H.-J., THAI, C. D. T. Outage performance of cognitive radio networks under outage constraint of multiple primary users and transmit power constraint of secondary user. In Proceedings of the Fifth International Conference on Ubiquitous and Future Networks (ICUFN). 2013, p. 631-635. DOI: 10.1109/ICUFN.2013.6614895

[25] FCC. Spectrum policy task force report, TR 02-155, Federal Communications Commission, 2002.

\section{About the Authors...}

Chanchal Kumar DE (corresponding author) was born in Bankura, India, in 1981. He received the Ph.D. degree in 2015. He is currently Assistant Professor in Electronics and Instrumentation Engineering department at Dr. B. C. Roy Engineering College (BCREC), Durgapur, India. His research interests include cooperative relay networks, OFDM technology, cognitive radio networks.

Sumit KUNDU was born in Durgapur, India, in 1970. He received his B.E. (Hons.) degree in Electronics and Communication Engineering in 1991 from NIT, Durgapur, India and M.Tech. degree in Telecommunication Systems Engineering and Ph.D. in Wireless Communication Engineering from IIT Kharagpur, India, respectively. He is now a professor and $\mathrm{Ph} . \mathrm{D}$ supervisor. His research interests include radio resource management in wireless networks, Wireless Ad Hoc and sensor networks, and Cognitive Radio Networks. 